\title{
Income Projections for Climate Change Research: A Framework Based on Human Capital Dynamics*
}

\author{
Jesús Crespo Cuaresma ${ }^{\dagger}$ \\ WU, IIASA, WIC and WIFO
}

\begin{abstract}
The quantitative assessment of the global effects of climate change requires the construction of income projections spanning large time horizons. Exploiting the robust link between educational attainment, age structure dynamics and economic growth, we use population projections by age, sex and educational attainment to obtain income per capita paths to the year 2100 for 144 countries. Such a framework offers a powerful, consistent methodology which can be used to study of future environmental challenges and to address potential policy reactions.
\end{abstract}

${ }^{*}$ The authour would like to thank Rob Dellink, Leiwen Jiang, Samir KC, Volker Krey, Elmar Kriegler, Marian Leimbach, Wolfgang Lutz, Brian O’Neill, Keywan Riahi, Detlef van Vuuren and two anonymous referees for extremely valuable input and discussions on earlier drafts of this manuscript.

†World Population Program, International Institute of Applied Systems Analysis (IIASA), Wittgenstein Centre for Demography and Global Human Capital (WIC), Department of Economics, Vienna University of Economics and Business (WU) and Austrian Institute for Economic Research (WIFO). Welthandelsplatz 1, 1020 Vienna, Austria, Email: jcrespo@wu.ac.at. 


\section{Introduction}

The development of socio-economic projection scenarios plays a central role in the assessment of climate change impacts, as well as in the design of policy responses. Scenario building facilitates the interdisciplinary research efforts that appear necessary for understanding the feedback mechanisms between climate impacts and socio-economic conditions. Recent proposals for climate research projection scenarios build on defining matrix structures where the interplay of climate signals and socio-economic developments is explicitly emphasized (see for example Van Vuuren et al. (2012)). To the extent that they are a central determinant of adaptation and mitigation, income developments appear as a particularly important component of such projection exercises, as highlighted in Van Vuuren et al. (2014) and O'Neill et al. (2014) for the case of the recent Shared Socioeconomic Pathways (SSPs).

The aim of this paper is to present a modelling framework to create scenarios for GDP and GDP per capita that can be used as an input in integrated assessment modelling and other climate research applications which require income per capita projections in the very long run. Our projection model has several advantages that make it particularly well suited to serve as an instrument in climate change impact assessments at the global level. On the one hand, its underlying structure, based on a standard macroeconomic production function with labour input differentiated by age and education attainment level, is simple and easy to communicate. On the other hand, since the GDP projections are driven by human capital dynamics (defined as the change of population by sex, age and educational attainment), the specification proposed can be combined with the population projections developed in the context of the SSPs by KC and Lutz (2014) in a straightforward manner. In addition to the population projection input, the model is calibrated by setting assumptions on physical capital investment, cross-sectional convergence and the developments in technological progress which are not related to human capital. The combination of the income projection method presented in this contribution with the population projection instruments described in KC et al. (2010) and used in KC and Lutz (2014) constitutes a self-contained and comprehensive framework to develop quantitative scenarios for assessing long-run environmental challenges. As such, it is thus designed to serve as a quantitative tool for assessing climate research questions related to the interplay of climate and socioeconomic systems. Furthermore, the relative simplicity of the approach makes it particularly suited to be adapted to other applications in social and environmental sciences.

From a theoretical point of view, the modelling strategy behind the projection exercise proposed in this paper builds upon a broad literature which emphasizes the role of human capital as a driver of economic growth (see for example Miller (1967) for a discussion on the role of education as a driver of economic growth in the history of economic thought). Early econometric contributions assessing the link between educational attainment and economic growth using large samples of countries tend to treat human capital as a standard input of production (see Mankiw et al. (1992)) whose accumulation affects income per capita in a similar fashion as physical capital accumulation does in earlier exogenous models of economic growth. The effect of education on labour productivity, a robust empirical stylized fact at the microeconomic level, justifies such an approach. Moving away from such an interpretation 
as a production factor, several contributions recognize the central role played by the stock of human capital (as opposed to the rate of accumulation) as a catalyst of technological innovation and foreign technology adoption (see Nelson and Phelps (1966) and Benhabib and Spiegel (1994)). In terms of model specification, such a generalization of the theoretical setting leads to empirical specifications where the stock of human capital and its interaction with the distance to the global technological frontier are used as explanatory covariates in addition to human capital accumulation variables.

In spite of the empirical evidence supporting the link between education and income at the macroeconomic level, very few contributions have used education as a basis for long-run out-of-sample economic growth projections. The lack of comparable data on educational attainment projections for broad sets of countries explains, at least partly, this gap in the literature. The information contained in age-structured population projections, which are available globally, has however been utilized to construct income forecasts (see Bloom et al. (2007) or Lindh and Malmberg (2007)). Recently, the availability of new global data of population by age, sex and educational attainment (see Lutz et al. (2007)) has enabled the integration of these branches of the literature and the investigation of the effect that the distribution of education across age groups exerts on economic growth. The results in Lutz et al. (2008) or Crespo Cuaresma and Mishra (2011) show that income growth differences across countries and over time can be better predicted if the age dimension of human capital is incorporated in the modelling framework.

Using a global dataset that spans the last four decades, in this contribution we estimate econometric models which capture the different aspects of the link between human capital and income growth highlighted in the literature. We combine such an estimated model with the projections of population by age, sex and educational attainment developed by KC and Lutz (2014) for practically all countries of the world to the year 2100. These population projections, in turn, have been designed to mimic the different qualitative scenarios (SSPs) which serve as a unifying frame in order to assess the impacts of climate change and the role played by adaptation and mitigation policies in counteracting its negative consequences.

The choice of a specification for the aim of projecting income per capita over very long horizons encounters a trade-off between expanding the model to include the manifold of factors affecting income and incorporating the uncertainty about the future trajectories of such determinants over the projection horizon. By concentrating on human capital dynamics, the model chosen tries to strike a balance between these two forces. Although the modelling framework employed in the projection exercise abstracts from some important economic growth determinants, comparable specifications to the one used in this contribution have been often used in the literature (see Benhabib and Spiegel (1994), Benhabib and Spiegel (2005), Lutz et al. (2008) or Barro and Lee (2013), just to name a few). The modelling approach has the advantage of using as an input education-structured population data, whose persistent dynamics allow for relatively precise and well understood projections. This contribution is framed in the context of research efforts aimed at providing economic growth projections for climate research based on the SSP storylines, thus complementing the reference projections provided by Chateau et al. (2014) for use in integrated assessment models (see also Leinbach et al. (2014) for an alternative 
complementary approach based on GDP aggregated at the level of world regions).

The paper is organized as follows. Section 2 presents the econometric model used to obtain the projections of GDP per capita. Section 3 summarizes the set of assumptions implied by each one of the SSPs, which define our projection scenarios. Section 4 presents the results of the income projection exercise, concentrating on the dynamics of world GDP per capita and the characteristics of each scenario in terms of the distribution of income per capita across countries. Finally, section 5 concludes.

\section{The Modelling Framework: Education, Age Struc- ture and Economic Growth}

The income projection model is based on a simple aggregate production function with heterogeneous labour input, which is differentiated by educational attainment (no education, primary, secondary and tertiary) and age group (younger and older workers, defined by a cut-off age of 35 years),

$$
Y_{i t}=A_{i t} K_{i t}^{\alpha} \prod_{j=0}^{3} \prod_{k=1}^{2} L_{i, j k t}^{\beta_{j k}}
$$

where $Y_{i t}$ is total output in country $i$ at time $t, A_{i t}$ refers to total factor productivity (TFP), $K_{i t}$ denotes the capital stock and $L_{i, j k t}$ corresponds to the labor input in age group $k$ ( $k=1,2$ denoting the younger and older age group) with educational attainment $j$ (from $j=0$ no education to $j=3$ some tertiary education level attained). Given the high correlation in the within-country dynamics of educational attainment by age group, there is a trade-off between using a low aggregation level in the age dimension and obtaining precise estimates of the model parameters. The potential multicollinearity problem caused by the co-movement of population by educational attainment calls therefore for the use of a small group of age groups in the specification of the economic growth regressions. The results in Lutz et al. (2008) indicate that the use of two broad age groups appears sufficient to explain global economic growth trends once the education dimension is added to the model. Given the persistence of population dynamics, the results of the estimation and projection exercise are not strongly affected by changing the age threshold that defines the older and younger group within the interval $(30,45)$. We also performed a principal components analysis exercise on the education data by age group which confirmed that the average level of education and the difference between older and younger age groups appears sufficient to summarize the variation of each one of the human capital variables over time and across countries.

Such a specification as the one given by equation (1) implies that the growth rate of total output depends on the growth rate of each one of the factors of production (TFP, the capital stock and each one of the population groups by age and educational attainment level),

$$
\Delta \log Y_{i t}=\Delta \log A_{i t}+\alpha \Delta \log K_{i t}+\sum_{j=0}^{3} \sum_{k=1}^{2} \beta_{j k} \Delta \log L_{i, j k t} .
$$


In addition, in the spirit of the Nelson-Phelps paradigm (see Nelson and Phelps (1966) and Benhabib and Spiegel (1994)), we assume that the growth rate of total factor productivity depends on three different factors:

(i) the distance to the technology frontier (as approximated by the average income per capita level of the country), which reflects (conditional) income convergence dynamics,

(ii) the stock of human capital in the country (measured as the ratio of population with different educational attainment levels to total population), which is used to proxy for the technology innovation potential of the economy and

(iii) the interaction between income per capita and the ratio of population with different educational attainment levels to total population, which accounts for technology adoption as a driver of income convergence.

The technology adoption argument suggests that countries which are further away from the technological frontier profit more than proportionally from investment in human capital, since an educated labour force accelerates the process of catching up with technology advancements. In our specification, we employ the approximation used by Benhabib and Spiegel (1994) and use income per capita instead of total factor productivity to define the gap between the current technology level of an economy and that of the technology frontier. Such a choice can be justified using different arguments: (a) income per capita and total factor productivity are highly (positively) correlated (see for example the results in Hall and Jones (1999)); (b) the inclusion of income per capita instead of total factor productivity relates our model to the large majority of empirical studies aimed at explaining income growth differences across countries, where conditional convergence is accounted for by including initial income per capita as an additional explanatory variable; (c) the qualitative implications of models including total factor productivity instead of income per capita do not differ substantially, as can be seen by comparing the results in Benhabib and Spiegel (1994) to those in Benhabib and Spiegel (2005).

This implies that the specification of the growth rate of total factor productivity in equation (2) can be written as

$$
\Delta \log A_{i t}=\delta \log \frac{Y_{i t}}{L_{i t}}+\sum_{j=1}^{3} \theta_{j} \sum_{k=1}^{2} \frac{L_{i, j k t}}{L_{i t}}+\sum_{j=1}^{3} \phi_{j} \log \frac{Y_{i t}}{L_{i t}} \sum_{k=1}^{2} \frac{L_{i, j k t}}{L_{i t}} .
$$

Such a modelling strategy implies that education plays the role of directly increasing labor productivity through acquired skills (an effect which is related to the fact that human capital is a direct input of the production function given by equation (1)) and of enabling the creation and adoption of new technologies (through the effects summarized in equation (3)), thus increasing economic growth by affecting the growth rate of total factor productivity. This setting, combining equations (2) 
and (3), leads to an econometric specification which is given by

$$
\begin{aligned}
\Delta \log Y_{i t}= & \alpha \Delta \log K_{i t}+\sum_{j=0}^{3} \sum_{k=1}^{2} \beta_{j k} \Delta \log L_{i, j k t}+\delta \log \frac{Y_{i t}}{L_{i t}}+ \\
& +\sum_{j=1}^{3} \theta_{j} \sum_{k=1}^{2} \frac{L_{i, j k t}}{L_{i t}}+\sum_{j=1}^{3} \phi_{j} \log \frac{Y_{i t}}{L_{i t}} \sum_{k=1}^{2} \frac{L_{i, j k t}}{L_{i t}},
\end{aligned}
$$

where the growth rate of population as well as its composition in terms of age structure and educational characteristics have an effect of economic growth.

We use data on purchasing power parity adjusted GDP from the Penn World Table 7.0 (Heston et al. (2011)) and construct capital stocks using the perpetual inventory method making use of investment data from the same source. As in Barro and Lee (2013), the depreciation rate of $6 \%$ was assumed for the computation of the capital stock series. The IIASA/VID dataset (see Lutz et al. (2007), Lutz et al. (2008) or KC et al. (2010)) provides (partly reconstructed) historical data on agestructured educational attainment for most countries in the world and is used as a source for the human capital variables of the model. The estimates are obtained using a panel dataset comprising information which spans the period 1970-2010 at five-year intervals for 120 countries. The variables which are measured in growth rates refer to the change in the corresponding five-year period, while the variables measured in levels are evaluated at the first year of the five-year interval, so as to avoid potential endogeneity problems. In addition to the variables implied by the production function framework described, we expand our specifications to include also overall population growth as an extra regressor. The panel structure of the dataset allows us to include country fixed effects and 5-year period fixed effects in the specification. The former account for unobserved, time-invariant, country-specific geographical, institutional or cultural characteristics that affect economic growth, while the period effects capture global income growth shocks which are common to all countries in a given 5-year interval. We use robust clustered standard errors in our estimation to account for potential deviations of the standard assumptions concerning the behaviour of the error term within countries.

The estimates corresponding to different specifications based on the framework described above are presented in Table 1 . We start by estimating a specification that exclusively assesses the convergence dynamics to country-specific balanced growth paths(see column 1), then we expand the specification by including human capital accumulation as a driver of income growth, assuming that the effects of education take place exclusively through its effect on labour productivity (column 2). In column 3 we include innovation effects in the specification by assuming that the stock of human capital affects technology and in column 4 we expand this specification further by including technology adoption effects (through the interaction of income per capita and the human capital stock). In all specifications the parameter estimates for the growth rate of total population are not significantly different from unity, which implies that the coefficient estimates for the variables of population by age and educational attainment level presented in Table 1 can be interpreted as effects on income per capita. Column 5 presents the estimates of the model including only the population variables whose parameter estimates are statistically significant after carrying out general-to-specific model selection based on significance tests for 
individual variables. The negative parameter estimates for initial income and its interaction with the tertiary education variable indicate that the speed of income convergence to a country-specific equilibrium depends on the stock of highly educated workers, thus lending empirical support to the role of education as a catalyst of technology adoption. This result implies that, for the same level of tertiary education, poorer countries benefit more of human capital than richer ones. The speed of convergence, thus, depends on the stock of human capital and the estimation results indicate that it is higher for those countries with a more educated population, as the Nelson-Phelps hypothesis (as interpreted by Benhabib and Spiegel (1994)) would suggest.

The positive and significant parameters attached to the share of working age population with secondary and tertiary education are interpreted in the context of Benhabib and Spiegel (1994) as technology innovation effects. The interaction of age structure and educational attainment appears relevant in terms of productivity improvements (summarized in the parameters attached to the growth rate of the respective population groups), where growth in the older age group with primary education and the younger age group with secondary education are particularly relevant in the specification used. The estimates of this model are used to obtain the projections which are presented in the following section. 


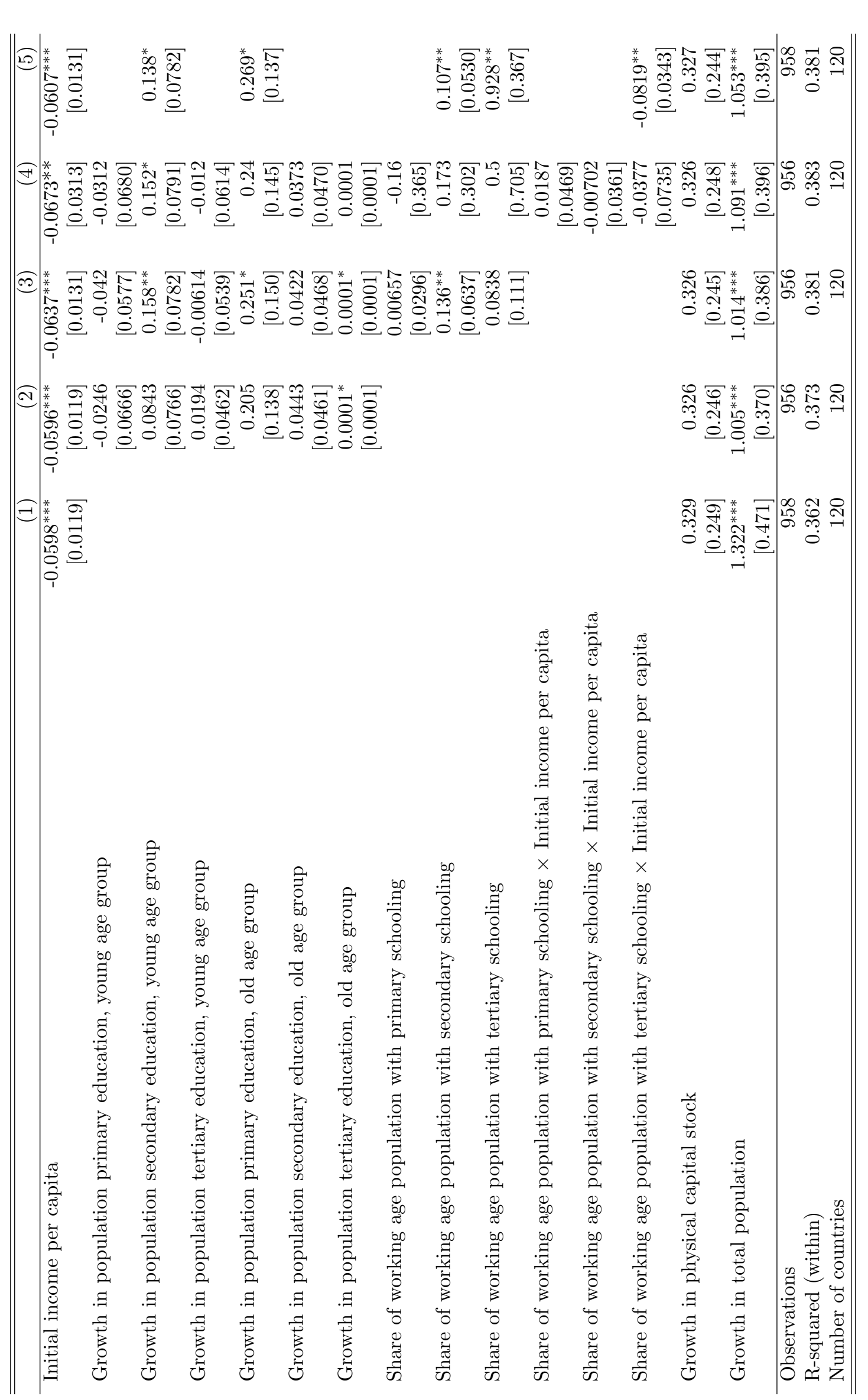

胥.

है 


\section{Shared Socio-Economic Pathways: Narratives, Assumptions and Projections of GDP}

O'Neill et al. (2014) and Van Vuuren et al. (2014) present the storylines that frame the scenarios used as Shared Socio-Economic Pathways (SSPs). They differ in terms of the challenges to mitigation and adaptation which are embodied in their narratives. Each one of the scenarios defined by the SSPs embodies several qualitative assumptions concerning the overall global economic growth and income convergence patterns in the projection period. Table 2 presents the qualitative description of each one of the SSP scenarios based on two dimensions: the growth rate of the technological frontier and the speed of income convergence across countries.

\begin{tabular}{llllll}
\hline \hline & SSP1 & SSP2 & SSP3 & SSP4 & SSP5 \\
\hline Frontier growth & Medium & Medium & Low & Medium & High \\
$\begin{array}{l}\text { Speed of Income } \\
\begin{array}{l}\text { Convergence } \\
\text { High }\end{array}\end{array}$ & Medium & Low & $\begin{array}{l}\text { Low Income Countries: Low } \\
\text { Medium Income Countries: Medium } \\
\text { High Income Countries: Medium }\end{array}$ & High \\
\hline \hline
\end{tabular}

Table 2: Shared Socio-Economic Pathways: Qualitative GDP growth assumptions by scenario

Projections of GDP and GDP per capita corresponding to the different SSP scenarios are obtained by combining assumptions on (a) the dynamics of population by age, sex and educational attainment, (b) the growth rate of total factor productivity which is not associated with human capital developments, (c) changes in the country fixed effects and (d) the behaviour of physical capital accumulation in the projection period.

Projections of population by age, sex and educational attainment for the different SSPs can be obtained from KC and Lutz (2014). The contribution by KC and Lutz (2014) complements the historical information contained in the IIASA/VID dataset with projections of age-structured population by educational attainment ranging until 2100. The projection scenarios put forward in KC and Lutz (2014) are based on the SSP narratives and can thus be directly used in the income projection model described in this work. Since conceptually the model proposed for GDP projections is based on the role played by human capital for economic development, the use of such population projections makes the modelling framework GDP optimal for the study of the interaction of demographic and economic variables, due to the selfcontainedness of the approach. In addition, in the logic of the model proposed to obtain GDP projections, the characteristics of the income paths implied by the SSPs presented in Table 2 are expected to be implied (at least partly) by the population dynamics in the scenarios constructed in KC and Lutz (2014).

The dynamics of total factor productivity embodied in our model are driven, on the one hand, by improvements in the human capital stock and income convergence forces (see equation (3)). On the other hand, the fact that our estimated model contains fixed time effects (in the form of 5 -year period dummies) implies that the specification allows for common global shocks to productivity, which can be 
interpreted as shifts of the global technology frontier which are independent of the human capital accumulation process. We build assumptions on the future of these shocks based on the examination of the past estimates of such global productivity shifts, which are quantified by the parameter estimates corresponding to the period dummies included in the model.

The specification proposed as a basis for the GDP projections contains country fixed effects, which are aimed at capturing unobserved country-specific, time-invariant characteristics of the economies modelled, as well as slow-changing institutional variables. Alternative scenarios about the behaviour of such fixed effects in the projection period allow us to recreate income convergence dynamics across countries which correspond to the different SSPs.

Concerning physical capital accumulation, we assume that the rate of growth of the capital stock will converge across countries over the coming decades. This hypothesis is maintained over all SSPs, since our projection exercise concentrates on human capital accumulation dynamics as a driver of income growth. Other assumptions concerning the dynamics of physical accumulation can be integrated in the modelling framework in a straightforward manner and deviations from the scenario settings are discussed in the robustness session below.

To summarize, we obtain projections for the five SSPs by combining the population projections for each pathway provided by KC and Lutz (2014) with the calibration of SSP-specific paths of global technological progress and of cross-country income convergence speed as implied by the convergence of the estimated fixed effects. Table 3 presents the quantitative assumptions used for the projections corresponding to each SSP. The convergence of fixed effects ("institutional convergence") is calibrated by defining the year at which all estimated fixed effects are assumed to converge to the value of the fixed effect estimated for the US. The growth rate of capital is assumed to converge to $4 \%$ per annum in the year 2100 . This value was obtained as the estimated unconditional expectation of physical capital growth in a panel autoregressive model using the available historical data and can thus be loosely interpreted as the common long-run equilibrium in terms of physical capital accumulation. In particular, using the available data we estimated the model $\Delta \log K_{i t}=\rho_{0}+\rho_{1} \Delta \log K_{i t-1}+\nu_{i t}$, where $\nu_{i t}$ is assumed to be a white noise disturbance term. The unconditional expectation is obtained as $\hat{\rho}_{0} /\left(1-\hat{\rho}_{1}\right)$, where $\hat{\rho}_{0}$ and $\hat{\rho}_{1}$ are the corresponding parameter estimates.

\begin{tabular}{lcc}
\hline \hline & $\begin{array}{c}\text { Technology frontier growth } \\
\text { (not human capital driven) }\end{array}$ & Institutional convergence by year ... \\
\hline SSP1 & $0.35 \%$ per year & 2180 \\
SSP2 & $0.3 \%$ per year & 2250 \\
SSP3 & $0.3 \%$ per year & No convergence \\
SSP4 & $0.3 \%$ per year & 2250 \\
SSP5 & $0.4 \%$ per year & 2130 \\
\hline \hline
\end{tabular}

Table 3: Shared Socio-Economic Pathways: Assumptions on technology frontier and institutional convergence by scenario

It should be mentioned that our decision to keep physical accumulation dynamics identical across scenarios rests upon the observational equivalence of different tra- 
jectories of technology and physical capital accumulation. In our framework, we can create identical projection paths based on alternative scenarios for capital and technology (say high growth of the capital stock and low technology growth versus low growth of the capital stock and high technology growth). As a result, when they are jointly specified, capital and technology dynamics are not uniquely identified in the scenarios and can be reinterpreted if some particular capital-to-income ratios were used to anchor the projections. This exogenous projection settings are thus in principle jointly defined for both physical capital and technology. In order to simplify the calibration of the projection, we decided to fix the capital dynamics across scenarios and base them on the observed global convergence trends of physical capital growth. This assumption, however, can be relaxed accordingly just by interpreting part of the dynamics of technology as physical capital changes without that affecting our results quantitatively.

Using the assumptions described above, GDP and GDP per capita growth projections were obtained for 144 countries (see Appendix for the list) and all five scenarios. Some countries - in particular emerging economies - only had available data for one of the five-year periods in the estimation sample. They were therefore not included in the estimation but we constructed GDP projections for them, using fixed effects based on economies with a similar level of economic development. The projected paths of income growth were further smoothed using a simple moving average filter centered in the corresponding projection year with a window of 10 years, so as to avoid sudden jumps in GDP and GDP per capita, in particular at the beginning of the projection exercise.

\section{Income Projections to 2100: An Overview}

In this section we give an overview of the main features of the SSP projections and we concentrate on the dynamics of GDP per capita. The projection data for GDP and GDP per capita obtained using the methodology proposed in this study can be retrieved from IIASA's SSP Database (https://secure.iiasa.ac.at/web-apps/ ene/SspDb/dsd?Action=htmlpage\&page=about). We start by assessing the dynamics of world income by scenario and then discuss the cross-country structure of the projections in terms of income convergence across SSPs.

\subsection{World Income Dynamics}

Figure 1 presents the average GDP per capita at the world level implied by the five scenarios, together with historical data ranging back to 1980 . In the year 2100, the scenarios span average world income per capita levels that range between approximately 20,000 int. \$ (basis year 2005) for SSP3 and roughly 100,000 int \$ for SSP5. The aggregated income projections resulting from the exercise imply substantial increases in income per capita for SSP5 and SSP1, while the dynamics of SSP2 are roughly in line with the historical trend. The SSP3 and SSP4 scenarios result on stagnating GDP per capita figures from mid-century onwards. The average growth rate of world income per capita over the full projection period ranges between $0.8 \%$ per year for SSP3 and 2.6\% per year in SSP5. Since the specification used implies 
convergence to country-specific equilibria, this leads to the fact that the projected rates of increase in GDP per capita for all scenarios are larger in the first part of the century than in later projection years. The additional convergence dynamics in terms of educational attainment which are embodied in the population projections by KC and Lutz (2014), together with the exogenous assumptions on physical capital accumulation and technology shocks, reinforce such an effect.

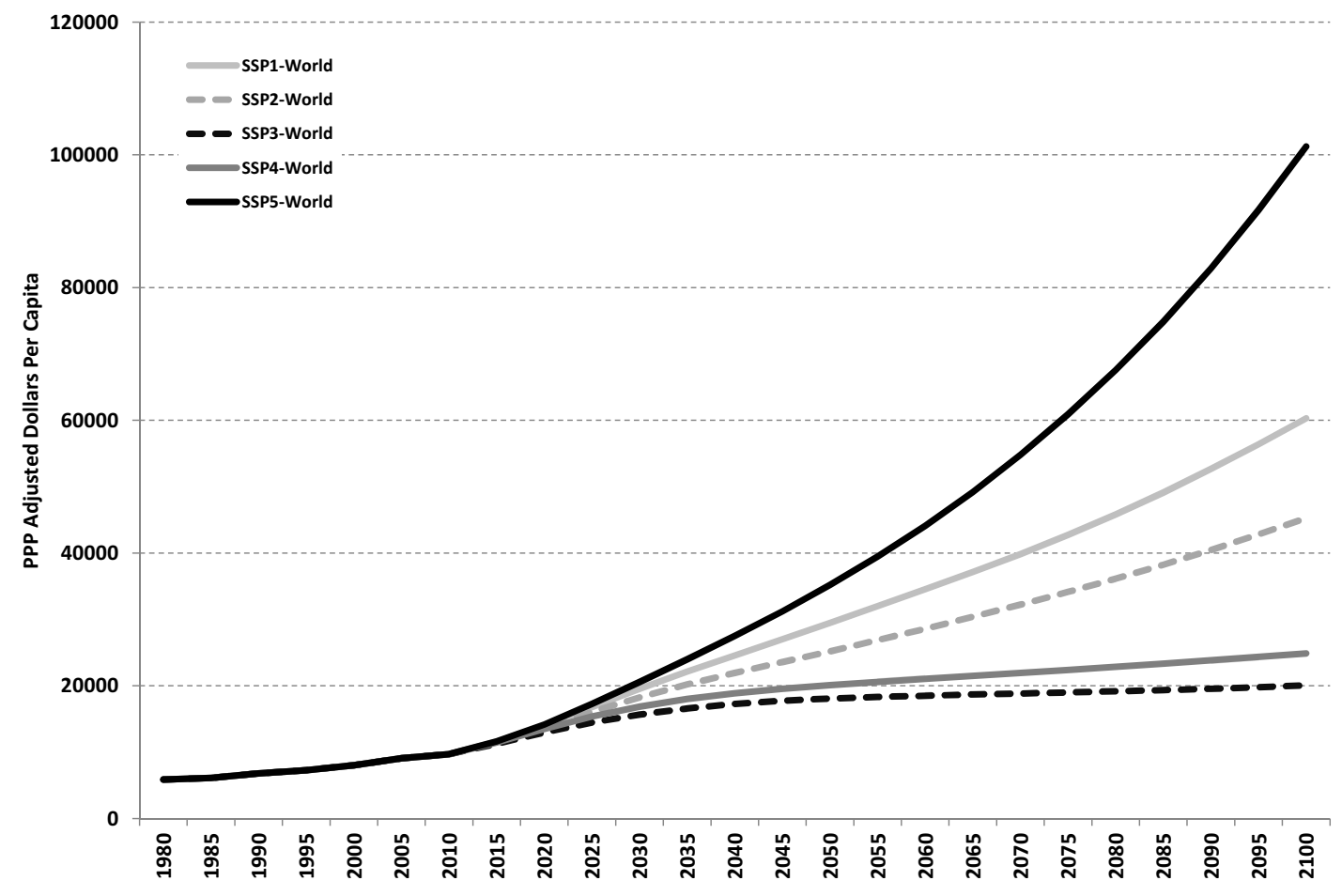

Figure 1: Projections of GDP per capita, world

The importance of human capital dynamics in shaping future income at the world level can be easily grasped by comparing GDP per capita under the SSP2 and the SSP4 scenarios. As can be seen in Table 3, both of these scenarios share the same overarching assumptions in terms of physical capital accumulation, institutional convergence and the growth of technology which is not caused by human capital accumulation. The average income differences for world GDP per capita between these scenarios can thus be interpreted as being mostly driven by the effect of human capital accumulation on labour productivity, innovation and the adoption of foreign technologies. The SSP4 scenario refers to a world of divergence and strong inequality both between and within countries, whose human capital dynamics lead to a polarized distribution of educational attainment on the world by the end of the 21st century. The inability of poor economies to catch up with industrialized countries in terms of educational attainment materializes itself in global average GDP per capita levels which are significantly smaller in this scenario as compared to SSP2, in spite of the similarity of their assumptions concerning the development of other economic growth determinants different from human capital. 


\begin{tabular}{cccccc}
\hline \hline & Period & World & $\begin{array}{c}\text { High income } \\
\text { countries }\end{array}$ & $\begin{array}{c}\text { Middle income } \\
\text { countries }\end{array}$ & $\begin{array}{c}\text { Low income } \\
\text { countries }\end{array}$ \\
\hline \multirow{3}{*}{ SSP1 } & $2010-2040$ & $3.2 \%$ & $1.7 \%$ & $4.8 \%$ & $4.1 \%$ \\
& $2040-2100$ & $1.5 \%$ & $1.3 \%$ & $1.5 \%$ & $2.7 \%$ \\
& $2010-2100$ & $2.1 \%$ & $1.4 \%$ & $2.6 \%$ & $3.2 \%$ \\
\hline \multirow{3}{*}{ SSP2 } & $2010-2040$ & $2.8 \%$ & $1.6 \%$ & $4.4 \%$ & $3.1 \%$ \\
& $2040-2100$ & $1.2 \%$ & $1.1 \%$ & $1.3 \%$ & $2.5 \%$ \\
& $2010-2100$ & $1.7 \%$ & $1.3 \%$ & $2.3 \%$ & $2.7 \%$ \\
\hline \multirow{3}{*}{ SSP3 } & $2010-2040$ & $1.9 \%$ & $0.9 \%$ & $3.9 \%$ & $1.7 \%$ \\
& $2040-2100$ & $0.3 \%$ & $1.1 \%$ & $0.5 \%$ & $0.7 \%$ \\
& $2010-2100$ & $0.8 \%$ & $1.0 \%$ & $1.6 \%$ & $1.0 \%$ \\
\hline \multirow{3}{*}{ SSP4 } & $2010-2040$ & $2.2 \%$ & $1.6 \%$ & $3.8 \%$ & $1.9 \%$ \\
& $2040-2100$ & $0.5 \%$ & $1.1 \%$ & $0.5 \%$ & $1.5 \%$ \\
& $2010-2100$ & $1.1 \%$ & $1.3 \%$ & $1.6 \%$ & $1.7 \%$ \\
\hline \hline
\end{tabular}

Table 4: World GDP per capita: Projected growth rates by income group and by SSP scenario

\subsection{The Cross-Country Dimension of Income Projections}

In order to understand the implications of each one of the projection scenarios in terms of the distribution of income per capita across countries, we start by presenting in Figure 2 the (between-country) population-weighted Gini coefficient computed for the projected data corresponding to each SSP.

The degree of inequality in average income per capita across economies decreases systematically in the scenarios which are dominated by cross-country income convergence trends (SSP1, SSP2 and SSP5). On the other hand, SSP3 and SSP4 present a trend towards more income inequality across economies starting around 2030. Within the convergent scenarios, by the end of the projection horizon the lowest degree of inequality is obtained in SSP5, which reaches a Gini coefficient value of roughly 0.25 in 2010. The GDP per capita projections for SSP1 and SSP2 also lead to significant decreases of inequality across countries over the projection period, leading to Gini coefficients of 0.33 and 0.41 by 2100 , respectively. 


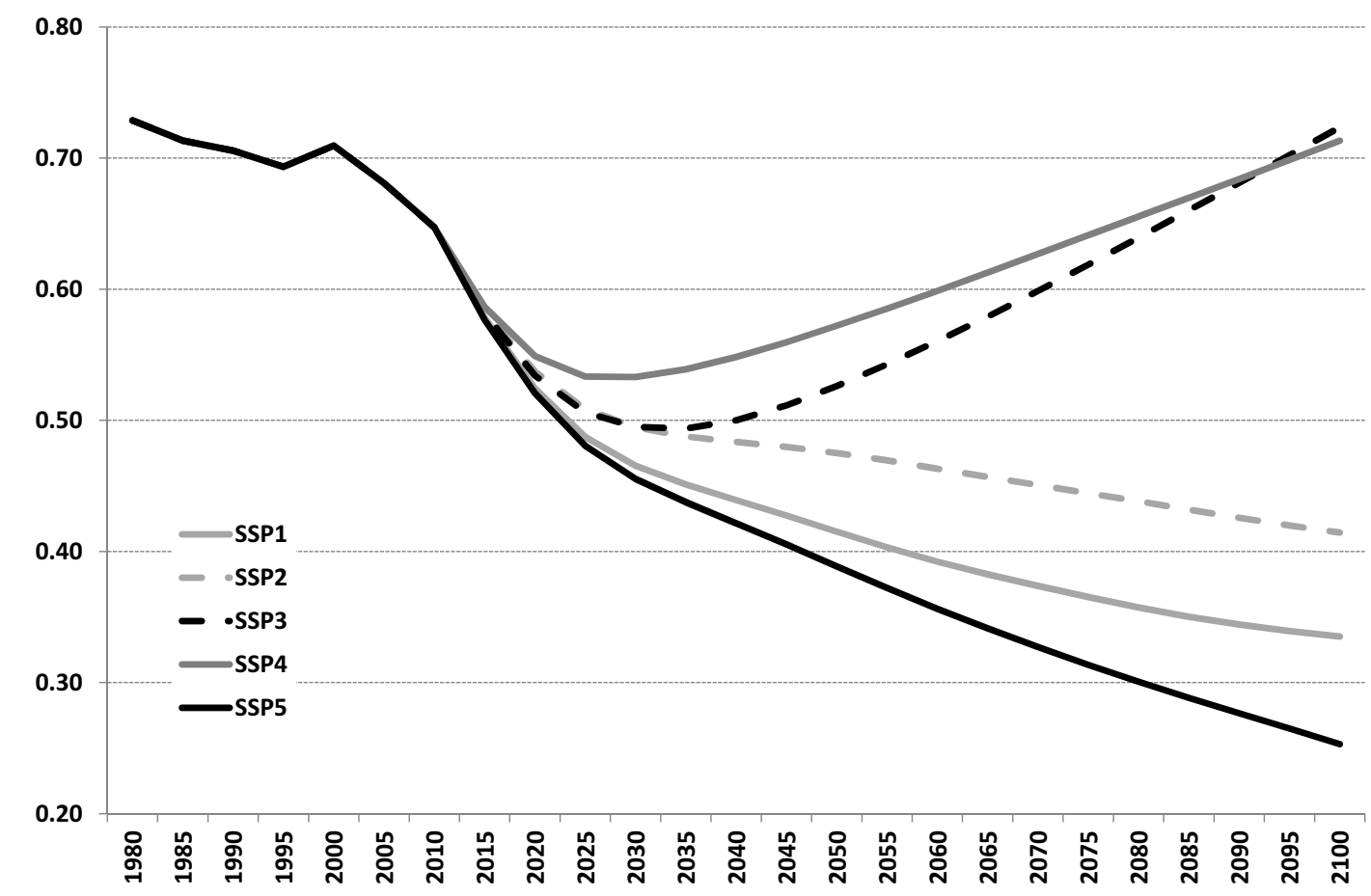

Figure 2: Population-weighted Gini coefficients, by SSP 

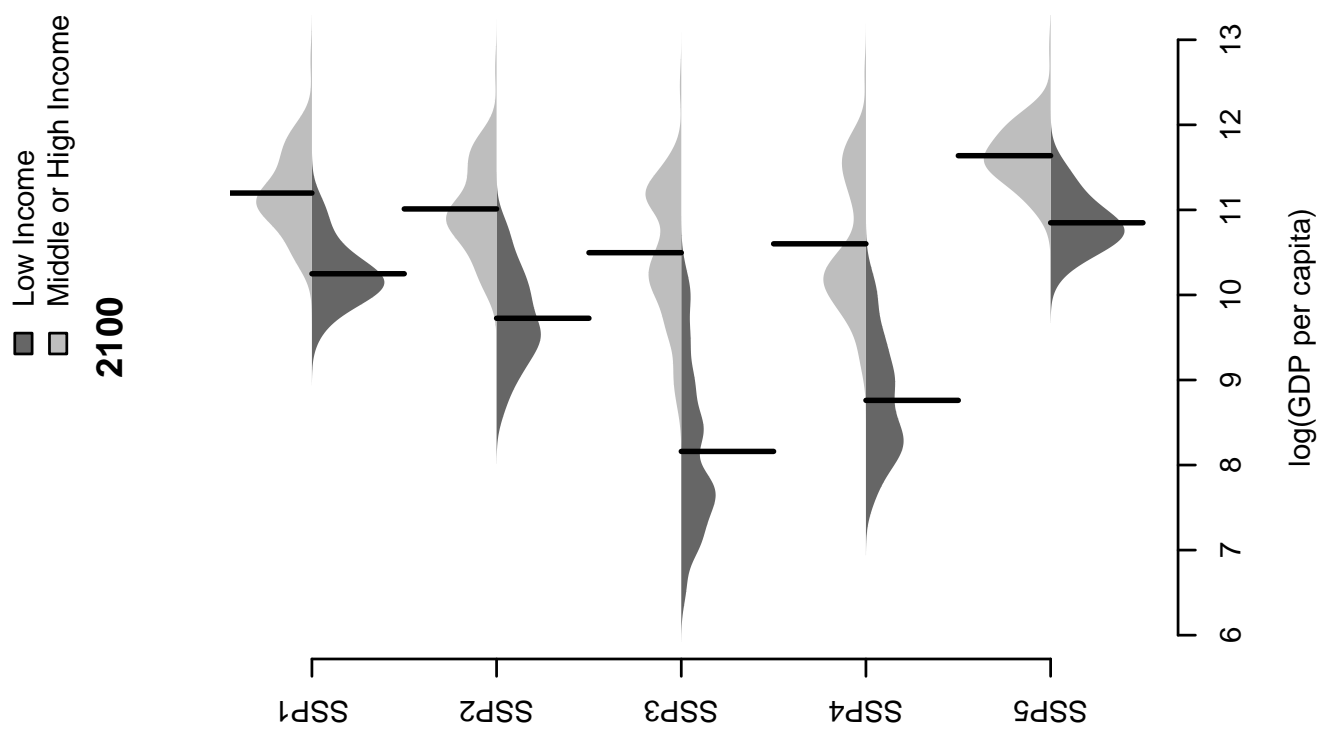

की

유
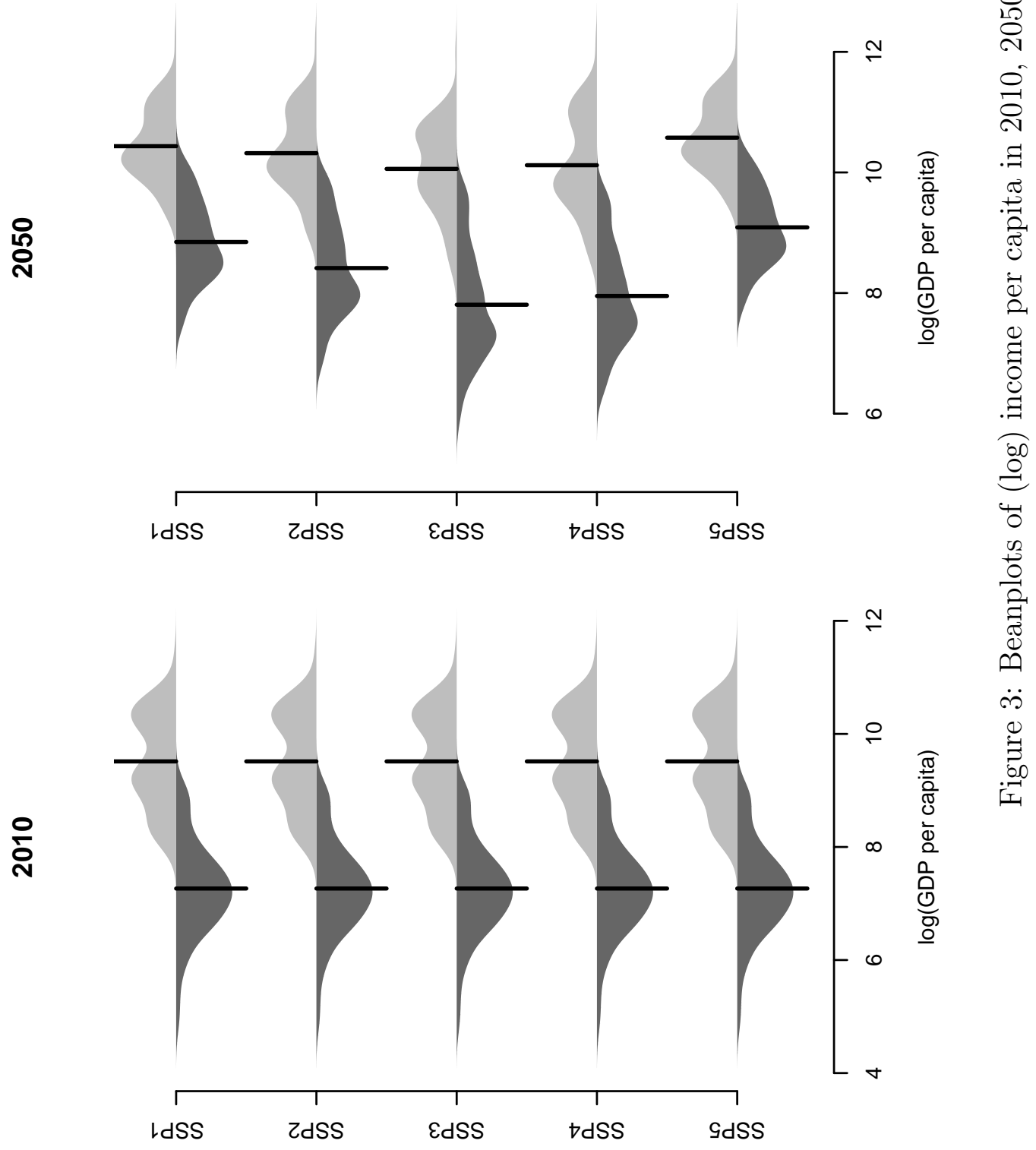
In order to assess graphically the changes in the distribution of income across countries implied by each one of the SSP projections, Figure 3 presents density plots (beanplots) which depict the distribution of (log) income per capita across projections in the years 2050 and 2100 for the groups of low income economies, as well as for that of middle and high-income countries (as of 2013). Beanplots (see Kampstra (2008)) can be interpreted as density trace plots and have clear advantages as compared to boxplots when it comes to representing graphically data whose distribution is multi-modal. The vertical lines in Figure 3 depict the means of each distribution, while the beans correspond to the corresponding density trace for the two subgroups. The convergence dynamics in income per capita across countries which are embodied in the SSP1, SSP2 and SSP5 scenarios are clearly recognizable in Figure 3 through the narrowing of the gap between the mean of two income distributions, together with the dynamics of the full distributions of income for low, middle and high income economies. The polarization of the income per capita distribution across countries in SSP3 and SSP4 is also visible in the density plots for the year 2100.

The different cross-country income convergence patterns in each scenario can be better understood with the aid of beta-convergence plots. Figure 4 shows, for each SSP scenario, a scatter plot depicting the level of (log) income per capita in 2010 (in the x-axis) against the cumulative growth of income between 2010 and 2100 (the log of the ratio of income per capita in 2100 to income in 2010) for each country. A negative correlation between the two variables indicates that income per capita tends to equalize across countries (income per capita in poorer economies in 2010 tends to grow more in the period 2010-2100 on average than in richer economies). The slope of the relationship relates to the speed of income convergence, with flatter relationships implying that the income gap across countries tends to persist for longer periods of time. The robust strong convergent dynamics embodied in the SSP5 and SSP1 scenarios as well as, to a lesser extent, in SSP2, can be seen in the beta-convergence plots of Figure 4. No significant income convergence across countries is observable in SSP3, while the weak income convergence structure in the SSP4 scenario is the result of polarized convergence to several long-run equilibria, as can be inferred from Figure 3. Such a polarized income per capita distribution in SSP4 emanates from the heterogeneity in terms of human capital accumulation which is embodied in the population projections for this scenario (see KC and Lutz (2014)), where the global high fertility/mortality versus low fertility/mortality divide is assumed to persist over the projection period. It should be noticed that, as opposed to the Gini indices presented in Figure 2, the observations in Figure 4 are not weighted by population. Cross-country convergence in mean income can thus be accompanied by increasing inequality once that population weights are added to the analysis, as is the case in our results for $\mathrm{SSP} 4$.

\subsection{Selected Robustness Checks}

Given the large number of alternative parameter calibration choices in the projection exercise, we performed a battery of robustness checks by changing the assumptions corresponding to the different scenarios. The interpretation of the scenario parametrization presented above, for instance, leads in some cases to very large changes in capital-to-output ratios over the projection horizon. The intuition be- 

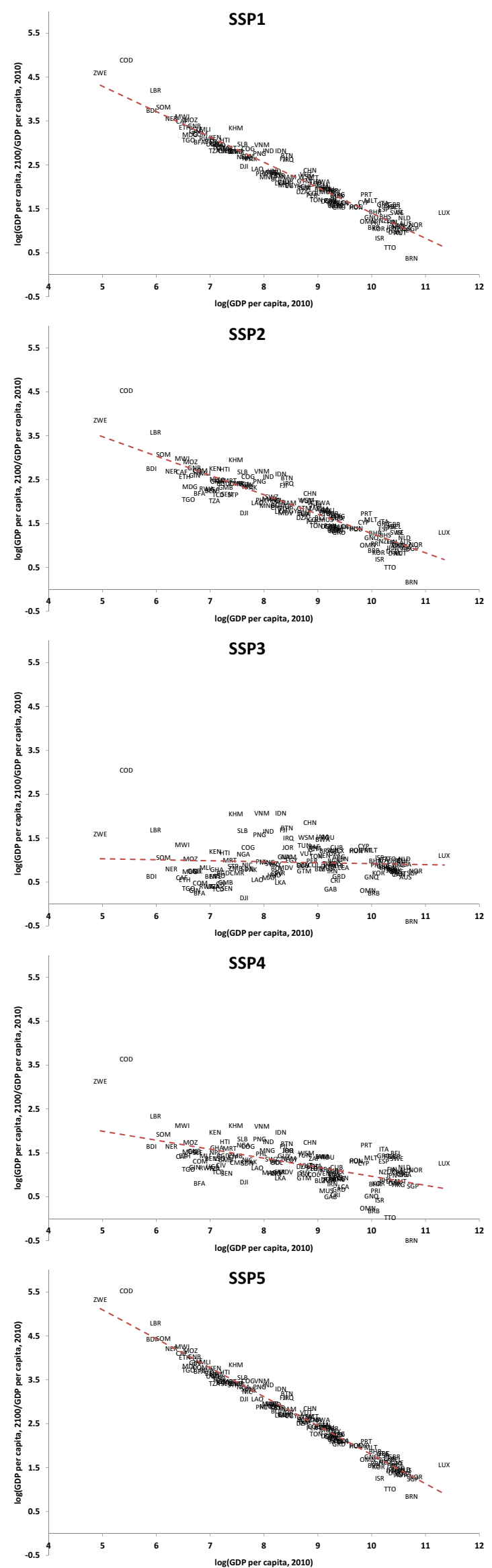

Figure 4: Projected growth in income (2010-2100) against (log) initial income, 2010 
hind the narratives of our projection scenarios is based on the existence of a balanced growth path for physical capital stocks sustained empirically by the historical experience in our sample of countries (that is, based on the convergence path observed in the data for physical capital accumulation). Alternatively, we also obtained simulations using within-country convergence of capital-to-output ratios as the relevant property for physical capital scenarios. In particular, we set the growth rate of the physical capital stock in period $t$ of the projection period equal to the growth rate of GDP in period $t-1$, so that in the long-run equilibrium the capital-to-output is constant. Such a change in the projection scenarios leads to a decrease in income per capita over the projection period but does not change the qualitative conclusions of the analysis. On average across all countries in the sample, this alternative assumption reduces GDP per capita in 2100 by $15 \%$ (standard deviation: $7.4 \%$ ) in SSP1 and by $15.5 \%$ (standard deviation: $5.9 \%$ ) in SSP3, for instance, as compared to our original projections. Detailed results of this alternative projection setting are available from the author upon request.

The nonlinear role that education plays on economic growth through its effect on technology adoption implies that it is not straightforward to decompose the effects of each one of the factors of production on long-run economic growth, as it would be the case in standard growth accounting exercises for the log-linear production function case. The importance of human capital dynamics in our scenarios can be grasped by performing counterfactual scenarios where the assumptions concerning educational attainment in a given SSP are imposed in a different one. Imposing the human capital dynamics of SSP3 on the SSP2 setting, for instance, leads to an average decrease in income per capita in 2100 of roughly $78 \%$ for low income economies (as implied by the log difference in GDP per capita, with a standard deviation of $27.9 \%$. This robustness check implies replacing human capital projections based on the so called Global Education Trend (GET) scenario with education dynamics which build on the assumption of constant enrolment rates (CER scenario, see KC and Lutz, 2014).

\section{Conclusions}

Projections of GDP and GDP per capita are important inputs for integrated assessment models aimed at measuring the effects of climate change. We propose to create such income scenarios by combining population projections by age, sex and educational attainment which have been developed in the context of recent scenariobuilding efforts for climate change research with a simple estimated economic growth model based on human capital dynamics. This modelling structure has the advantage of providing an internally consistent framework to simulate GDP per capita paths which correspond to the narratives put forward in the framework of the recently developed Shared Socioeconomic Pathways (see O'Neill et al. (2014) and Van Vuuren et al. (2014)).

This contribution is the first one, to our knowledge, to propose an econometric tool aimed at long-run income projection for climate change research which stresses the role of human capital dynamics. As such, several efforts to improve the modelling framework further and account for some of the potential weaknesses of the approach 
need to be carried out in the future. While the focus of the projection methodology on human capital dynamics lends parsimony to the approach, for many applications such a simplification may appear too far-fetched. This is particularly the case if trend breaks or disruptive short-lived events need to be accounted for in the projection period. Further research integrating a more systematic assessment of physical capital dynamics and institutional change should be able to expand the range of applications of this income projection tool.

The model put forward lends itself to applications beyond climate change research and provides a basis for further scenario-building exercises. The simplicity and flexibility of the specification used makes the method particularly attractive to assess research questions related to the macroeconomic behaviour of countries in the very long run when human capital dynamics are the core of the analysis. 


\section{References}

Barro, R.J. and Lee, J-W. (2013), A New Data Set of Educational Attainment in the World, 1950-2010, Journal of Development Economics 104, 184-198

Benhabib J. and Spiegel M. (1994), The Role of Human Capital in Economic Development: Evidence from Aggregate Cross-Country Data, Journal of Monetary Economics 34, 143-173

Benhabib J. and Spiegel M. (2005), Human Capital and Technology Diffusion, in: Philippe Aghion and Steven Durlauf (ed.), Handbook of Economic Growth, Chapter 13, pages 935-966, Elsevier.

Bloom, D.E., Canning, D., Fink, G. and Finlay, J.E. (2007), Does Age Structure Forecast Economic Growth?, International Journal of Forecasting 23, 569-585.

Chateau, J., Dellink, J., Lanzi, E. and Magne, E. (2014), Long-Term Economic Growth and Environmental Pressure: Reference Scenarios for Future Global Projections Global Environmental Change, this issue.

Crespo Cuaresma, J. and Mishra, T. (2011), The Role of Age-Structured Education Data for Economic Growth Forecasts, Journal of Forecasting 30, 249-267

Hall, R.E. and C.I. Jones (1999), Why Do Some Countries Produce So Much More Output Per Worker Than Others? Quarterly Journal of Economics 114, 83-116

Heston A., Summers, R. and Aten, B. (2011), Penn World Table Version 7.0, Center for International Comparisons of Production, Income and Prices at the University of Pennsylvania, May 2011

Kampstra, P. (2008), Beanplot: A Boxplot Alternative for Visual Comparison of Distributions. Journal of Statistical Software 28, Code Snippet 1, 1-9

KC, S., Barakat, B., Goujon, A., Skirbekk, V., Sanderson, W. and Lutz, W. (2010), Projection of Populations by Level of Educational Attainment, Age, and Sex for 120 Countries for 2005-2050. Demographic Research 22, 383-472

KC, S. and Lutz, W. (2014), Demographic Scenarios by Age, Sex and Education Corresponding to the SSP Narratives, Population and Environment 35, 243-260

Kriegler, E., O’Neill, B.C., Hallegatte, S., Kram, T., Lempert, R., Moss, R. and Wilbanks, T. (2013), The need for and use of socio-economic scenarios for climate change analysis: A new approach based on shared socio-economic pathways, Global Environmental Change, forthcoming.

Leimbach, M., Kriegler, E., Roming, N. and Schwanitz, V. (2014), Future growth patterns of world regions - A GDP scenario approach, Global Environmental Change Global Environmental Change, this issue.

Lindh, T. and Malmberg, B. (2007), Demographically Based Global Income Forecasts Up To The Year 2050, International Journal of Forecasting 23, 553-567. 
Lutz, W., Goujon, A., K.C., S. and Sanderson, W. (2007), Reconstruction of Populations by Age, Sex and Level of Educational Attainment for 120 Countries for 1970-2000, Vienna Yearbook of Population Research , 193-235

Lutz, W., Crespo Cuaresma, J. and Sanderson, W. (2008), The Demography of Educational Attainment and Economic Growth, Science 319, 1047-1048

Mankiw, N.G., Romer, D. and Weil, D.N.W. (1992), A Contribution to the Empirics of Economic Growth, Quarterly Journal of Economics 107, 407-437

Miller, W.L. (1967), Education as a Source of Economic Growth, Journal of Economic Issues 1, 280-296

Nelson R. and Phelps E. (1966), Investment in Humans, Technological Diffusion and Economic Growth, American Economic Review, Papers and Proceedings 56, 69-75

O’Neill, B.C., Kriegler, E., Riahi, K., Ebi, K.L.E., Hallegatte, S., Carter, T.R., Mathur, R. and van Vuuren, D.P. (2014), A New Scenario Framework for Climate Change Research: The Concept of Shared Socioeconomic Pathways, Climatic Change 122, 387-400

Van Vuuren, D.P., Riahi, K., Moss, R., Edmonds, J., Thomson, A., Nakicenovic, N., Kram, T., Berkhout, F., Swart, R., Janetos, A., Rose, S.K. and Arnell, N. (2012), A Proposal for a New Scenario Framework to Support Research and Assessment in Different Climate Research Communities, Global Environmental Change 22, 21-35

Van Vuuren, D.P., Kriegler, E., O’Neill, B.C., Ebi, K.L.E., Riahi, K., Carter, T.R., Edmonds, J., Hallegatte, S., Kram, T., Mathur, R. and Winkler, H. (2014), A New Scenario Framework for Climate Change Research: Scenario Matrix Architecture, Climatic Change 122, 373-386 


\section{Appendix}

\section{Fixed effect estimates}

The fixed effects of the estimated model are interpreted as the effect of (partly unobservable) time-invariant country-specific characteristics on economic growth. These include geographical, institutional and cultural factors. The different scenarios used in the projection exercise imply different dynamics of such effects over the projection period. In addition, the period fixed effects included in the model account for global economic growth shocks to all countries in the sample.

The estimated country fixed effects for the sample based on the period 1970-2010 are presented in Figure A.1 below and the period fixed effects in Figure A.2.

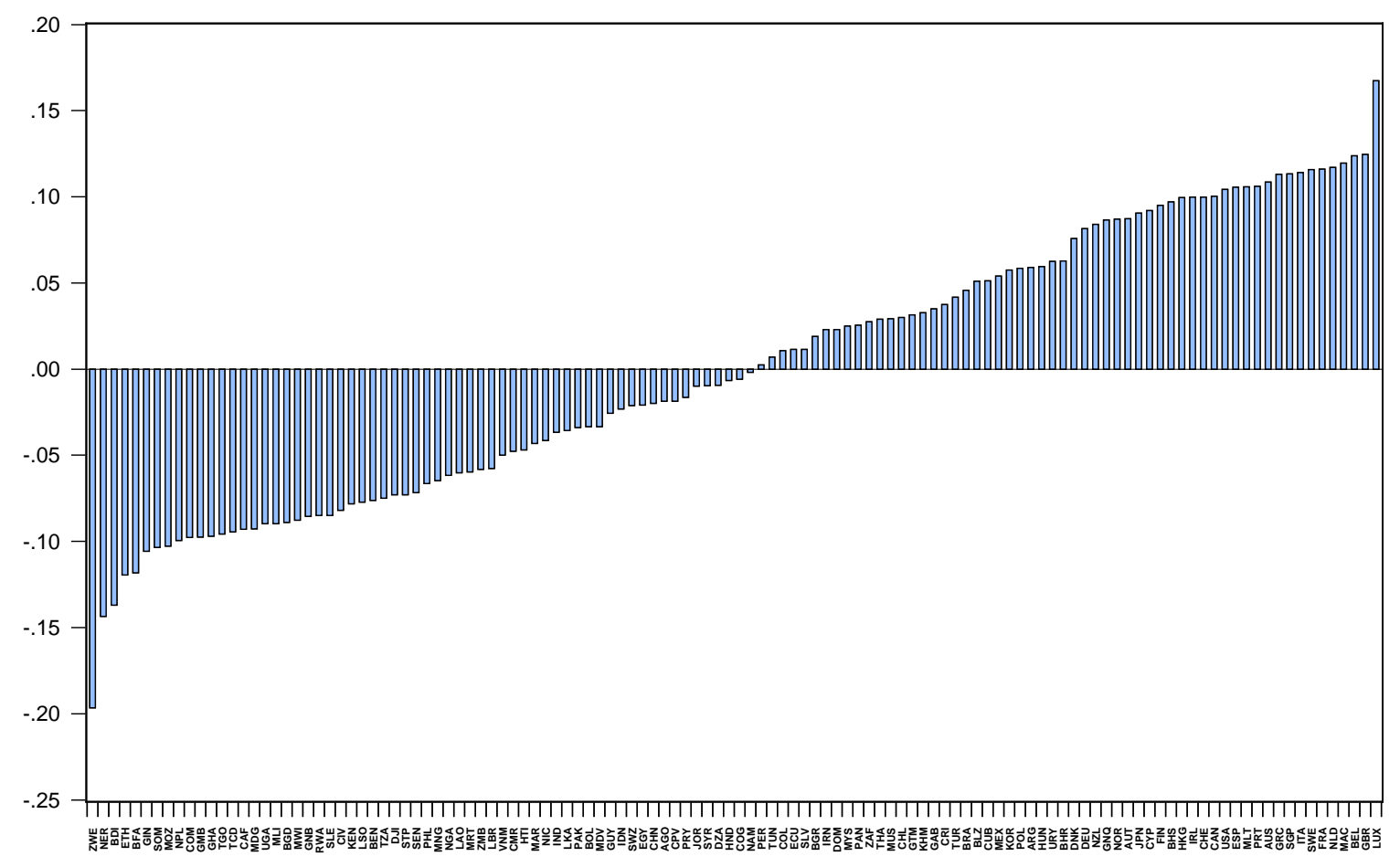

Figure A.1: Country fixed effect estimates 


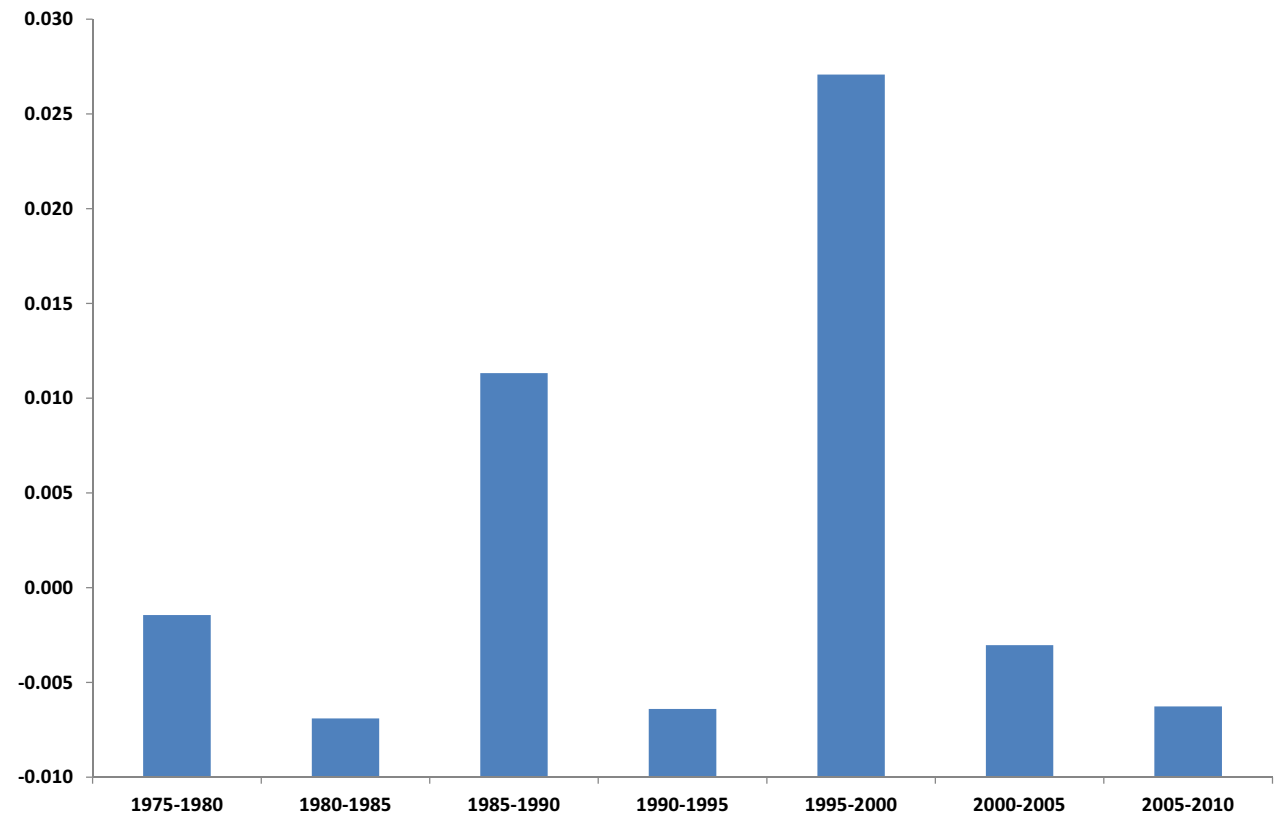

Figure A.2: Period fixed effect estimates 


\section{Countries in the Projection Exercise}

\begin{tabular}{|c|c|c|c|c|}
\hline Africa & America & Asia & Europe & Oceania \\
\hline Algeria & Argentina & Bahrain & Austria & Australia \\
\hline Benin & Bahamas & Bangladesh & Belgium & Fiji \\
\hline Botswana & Barbados & Bhutan & Bulgaria & New Zealand \\
\hline Burkina Faso & Belize & Brunei & Denmark & Papua New Guinea \\
\hline Burundi & Bolivia & Cambodia & Finland & Samoa \\
\hline Cameroon & Brazil & China & France & Solomon Islands \\
\hline Cape Verde & Canada & Cyprus & Germany & Tonga \\
\hline Central African Republic & Chile & Hong Kong & Greece & Vanuatu \\
\hline Chad & Colombia & India & Hungary & \\
\hline Comoros & Costa Rica & Indonesia & Iceland & \\
\hline Congo & Cuba & Iran & Ireland & \\
\hline Dem. Rep. Of Congo & Dominican Republic & Iraq & Italy & \\
\hline Cte d'Ivoire & Ecuador & Israel & Luxembourg & \\
\hline Djibouti & El Salvador & Japan & Malta & \\
\hline Egypt & Grenada & Jordan & Netherlands & \\
\hline Equatorial Guinea & Guatemala & Korea & Norway & \\
\hline Ethiopia & Guyana & Lao & Poland & \\
\hline Gabon & Haiti & Lebanon & Portugal & \\
\hline Gambia & Honduras & Malaysia & Romania & \\
\hline Ghana & Jamaica & Maldives & Spain & \\
\hline Guinea & Mexico & Mongolia & Sweden & \\
\hline Guinea-Bissau & Nicaragua & Nepal & Switzerland & \\
\hline Kenya & Panama & Oman & United Kingdom & \\
\hline Lesotho & Paraguay & Pakistan & & \\
\hline Liberia & Peru & Philippines & & \\
\hline Madagascar & Puerto Rico & Singapore & & \\
\hline Malawi & Saint Lucia & Sri Lanka & & \\
\hline Mali & St Vincent and the Grenadines & Syria & & \\
\hline Mauritania & Suriname & Thailand & & \\
\hline Mauritius & Trinidad and Tobago & Turkey & & \\
\hline Morocco & United States & Vietnam & & \\
\hline Mozambique & Uruguay & & & \\
\hline Namibia & Venezuela & & & \\
\hline \multicolumn{5}{|l|}{ Niger } \\
\hline \multicolumn{5}{|l|}{ Nigeria } \\
\hline \multicolumn{5}{|l|}{ Rwanda } \\
\hline \multicolumn{5}{|l|}{ Sao Tome and Principe } \\
\hline \multicolumn{5}{|l|}{ Senegal } \\
\hline \multicolumn{5}{|l|}{ Sierra Leone } \\
\hline \multicolumn{5}{|l|}{ Somalia } \\
\hline \multicolumn{5}{|l|}{ South Africa } \\
\hline \multicolumn{5}{|l|}{ Sudan } \\
\hline \multicolumn{5}{|l|}{ Swaziland } \\
\hline \multicolumn{5}{|l|}{ Tanzania } \\
\hline \multicolumn{5}{|l|}{ Togo } \\
\hline \multicolumn{5}{|l|}{ Tunisia } \\
\hline \multicolumn{5}{|l|}{ Uganda } \\
\hline \multicolumn{5}{|l|}{ Zambia } \\
\hline Zimbabwe & & & & \\
\hline
\end{tabular}

